\section{Thyroid cancer incidence}

SIR - As mentioned in two recent contributions to Scientific Correspondence $^{1,2}$, the increase in the number of reported thyroid cancer cases in Belarus, particularly in Gomel $^{3,4}$, could have arisen from the effect of better reporting, heightened awareness and screening. To help interpret the Belarus results, we estimated the magnitude of the effect of screening in a cohort of children treated at the Michael Reese Hospital by irradiation for benign head and neck diseases.

In 1974, newspapers in the United States were drawing attention to the excess of thyroid cancer among people two factors: (1) the intense screening efforts between 1974-79 detected early tumours and, therefore, fewer remained to be diagnosed later; and (2) screening was not as intense during subsequent years.

To reduce the effect of increased incidence from radiation exposure and thus isolate the effect of screening, a similar analysis was performed for the lowestdose group category (less than 50 centigray). In this group, the difference in the incidence of thyroid tumours before and after 1974 was even greater (see table).

Our results were based on examination of adults; Mettler et al. have

INCIDENCE OF THYROID CANCER AND NODULES

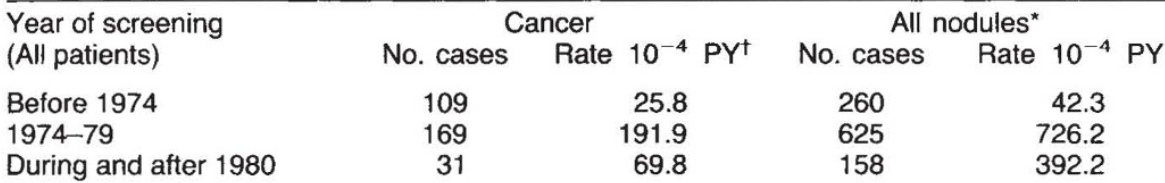

(Patients receiving $<50$ centigray)

$\begin{array}{lrrrr}\text { Before 1974 } & 18 & 14.0 & 40 & 19.7 \\ 1974-79 & 47 & 169.1 & 138 & 418.9 \\ \text { During and after 1980 } & 9 & 68.1 & 40 & 235.1\end{array}$

Patients were treated with external radiation for benign head and neck conditions at Michael Reese Hospital.

* Includes patients with malignant and benign tumours, as well as patients with thyroid nodules not surgically removed.

t Gender and age adjusted to a person aged 30 years. PY, person years.

treated with head and neck radiation. At the same time, Michael Reese Hospital began a recall and screening programme for more than 5,000 people who had received radiation treatment ${ }^{5}$. For 2,634 $(68.5 \%)$ patients who were 15 years of age or younger at the time of treatment (mean age 4.3 years) and for whom thyroid doses were computed, information about the occurrence of thyroid nodules was obtained from a screening examination at the hospital or from a questionnaire (A.B.S. et al., manuscript submitted). Surgically removed thyroid tumours reported in the questionnaires were histologically confirmed.

To evaluate the effect of screening, we compared incidence rates before the screening programme; during the most active period of screening (1974-79); and when the level of screening had subsided somewhat (after 1980). The 6-year period between 1974 and 1979 is most comparable, in terms of screening effects, to the period after the Chernobyl accident (1987-92). As seen in the table, the adjusted incidence rates were much greater during the 1974-79 period than before 1974 (7- and 17-fold for malignant and all thyroid nodules, respectively). These increases are of the order of those seen in Belarus. The decline in incidence rates seen after 1979 probably reflects reported $^{6}$ that $0.7 \%$ of a small sample of 5- and 10-year-olds living in contaminated and control settlements around. Chernobyl had palpable thyroid nodules, suggesting that even among young children, thyroid nodules occur frequently and can be detected by screening. Although our data should not be interpreted as showing that there is no effect of radiation from Chernobyl, they demonstrate that carefully controlled epidemiological studies are needed to understand the true impact of the accident.

\section{Elaine Ron}

Jay Lubln

Epidemiology and Biostatistics Program,

National Cancer Institute,

National Institutes of Health, Bethesda,

Maryland 20892, USA

Arthur B. Schneider

Division of Endocrinology and

Metabolism,

Humana and Michael Reese hospitals,

University of Illinois,

Chicago, Illinois 60616, USA

1. Beral, V. \& Reeves, G. Nature 359, 680-681 (1992).

2. Shigematsu, I. \& Thiessen, J. W. Nature 359, 681 (1992).

3. Kazakov, V. S. et al. Nature 359, 21 (1992)

4. Baverstock, K. et al. Nature 359, 21-22 (1992).

5. Baverstock, K. et al. Nature 359, 21-22 (1992).

5. Schneider, A. B. et al. Medicine 64, 1-15 (1985).
6. Mettler, F. A. et al. J. Am. med. Ass. 268, 616-619 Mettler,
(1992)
Ice-crystal growth and lectins

SIR - The C-type lectin found in rattlesnake venom can modify the growth habit of ice crystals in a manner similar to that previously thought unique to fish antifreeze proteins (AFP) $)^{1-3}$. Four main types of AFP have been identified in the blood sera of various teleost fishes which protect fish from freezing by depressing the freezing temperature of their body fluids non-colligatively. These proteins act by preferentially binding to the nonbasal planes of ice crystals, thereby modifying their growth habit to produce unique spicular, faceted structures rather than the usual smooth, non-faceted ones $^{1-3}$

Recently, we reported that one of the AFP types (II) found in three distantly related fish species (sea raven, smelt and herring) has a structure similar to the carbohydrate-recognition domain (CRD) of calcium-dependent (C-type) lectins ${ }^{4}$. This prompted us to determine whether these lectins possess AFP-like properties. To this end, we used the lectin isolated from the venom of the rattlesnake (Crotalus atrox). This lectin is a dimer with a $M_{\mathrm{r}}$ of 28,000 ; the native form appears aggregated into a larger multimeric structure ${ }^{5,6}$. Each monomer consists of a CRD. The lectin was purified from snake venom by affinity chromatography essentially following previously described methods ${ }^{5,6}$.

Initial studies with this protein using a Clifton nanolitre osmometer did not reveal any non-colligative freezing point depression at the resolution of the device. However, using an apparatus that allows microscope examination of the freezing interface during unidirectional freezing under controlled temperature conditions (directional solidification stage $^{7}$ ) we find that the lectin does possess properties that so far have been unique to antifreeze proteins ${ }^{1-3}$. Selected results of unidirectional freezing, with freezing velocities of $2.5 \pm 0.7 \mathrm{~mm} \mathrm{~min}^{-1}$ and temperature gradients of $15 \pm 1{ }^{\circ} \mathrm{C}$ $\mathrm{mm}^{-1}$, are shown in the figure.

The upper panel illustrates, in two ice crystals separated by a grain boundary, the planar ice-crystal morphology typical of pure water and aqueous solutions containing low concentrations of solutes. This particular result was obtained with a $0.71 \mathrm{mM}$ solution of $\mathrm{NaCl}$ in distilled water. A similar morphology was observed with distilled water; $2.5 \mathrm{mM}$ and $5 \mathrm{mM} \mathrm{CaCl}$; and $0.71 \mathrm{mM}, 2.5 \mathrm{mM}$ and $5 \mathrm{mM}$ lactose. The smooth nonfaceted morphology of the ice crystal suggests that the freezing interface is rough on an atomic or molecular scale, and that the growth rates are indepen- 